\title{
Analysis of BitTorrent on an Open Source VPN Technology to End User
}

\author{
Farok Azmat \\ Faculty of Information Technology and Quantitative Sciences \\ MARA University of Technology (UiTM) \\ 40450 Shah Alam, Selangor, MALAYSIA \\ Tel: 60-3-5521-1148 E-mail: farok@tmsk.uitm.edu.my \\ Md Ikhwan Hamdan \\ Faculty of Information Technology and Quantitative Sciences \\ MARA University of Technology (UiTM) \\ 40450 Shah Alam, Selangor, MALAYSIA \\ Tel: 60-3-5521-1148Ｅ-mail: zegxro@gmail.com \\ Kamaruzaman Jusoff (Corresponding author) \\ Yale University, Yale's CEO, Environmental Science Centre \\ 21 Sachem St., New Haven, CT 06511, USA \\ Tel: 20-3-432-1384Ｅ-mail: jusoff.kamaruzaman@yale.edu
}

\begin{abstract}
The local broadband provider Streamyx has imposed an in admitted practice of line throttling. As the result it affects the speed of BitTorrent download. To overcome the issue a procedure is to be found. Based on the fact that VPN is normally untouched by most ISP hence this technology is explored to be deployed on BitTorrent. Subsequently in this part of the article a further observation and analysis are done to supplement the previously rudimentary findings to gauge the effect of VPN circumventing the throttling. A final conclusion is thus deduced to find out whether this procedure is effective $24 / 7$ or it is bounded by the constraint of the normal working hours of the week i.e. Monday to Friday or otherwise beyond it.
\end{abstract}

Keywords: BitTorrent, Broadband, End user, File sharing, ISP throttling, Jaring, Open Source, OpenVPN, P2P, Peer-to-Peer, Proxy server, Streamyx, uTorrent, VPN

\section{Introduction}

The Malaysian broadband scenario has seen an in admitted throttling by Streamyx (Asohan, 2007 and Teoh, 2006). It was compounded after the Taiwan earthquake which disrupted the Malaysian international link and the throttling seems to stay indefinitely. To confirm this Zeropaid.com (2008) in its latest report has identified that Streamyx is one of the world ISPs offender of BitTorrent. Furthermore it is also mentioned that the strategy plan in which VPN is to be deployed on the BitTorrent download is shown as Figure 1 below.

$<<$ Figure 1. Location of VPN server $>>$

This figure shows the virtual path of VPN maneuvering from the US high speed backbone through the international line via Malaysia right up to the end user where the final leg is determined by the local ISP opted by the end user (TM News Release, 2007). However, it has to be realized that the server hardware must be placed at the region of high bandwidth such as to create the origin of the VPN tunnel. The server side must be ensured that no throttling is imposed at that point. The end user or client who is at the end of the tunnel will get the speed determined by the local ISP bandwidth as opted by the user. Finally, the overall arbitrary tunnel created by VPN is visualized as shown in the Figure 2 below.

$<<$ Figure 2. The arbitrary tunnel created by VPN $>>$ 
Figure 2 above shows that the origin of any download will start at the high speed backbone point whereas the user or client will be anywhere else as not confined to any particular location.

\section{Methods and materials}

\subsection{Server and client side}

On the high bandwidth backbone, server hardware has to be acquired while on the server machine the OpenVPN was installed. This OpenVPN has to be configured accordingly. A proxy server was also installed to suite each member of end user accordingly to its opted speed. On the other hand, for the Client side, the OpenVPN was installed where a certificate for the client was provided by the administration. A BitTorrent software client has to be setup up accordingly as given by the admin such as Proxy IP address number and Proxy Port number.

\subsection{Broadband speed}

Prior to doing the full analysis it is appropriate for the user to initially test the line speed using any of the speed tests available on the internet. There is one provided by Streamyx but at the time of this paper is prepared the site is unavailable (Figure 3). Fortunately, Jaring another local ISP has the facility and was used instead. Figure 4 shows the result of the local streamyx line done by Jaring.

$<<$ Figure 3. Streamyx speedometer speed test

(Source: http://202.188.95.52:8080/speedometer/)>>

$<<$ Figure 4. Speed test result given by Jaring ISP on Streamyx line.

(Source: http://www.jaring.my/service/broadband/index.cfm?cont=bandwidth_business) $>>$

Next, a second speed test is initiated. This time the speed test is done after the installation of VPN server at the foreign high speed backbone line. This test includes the speed from the international line to the end user. The result is shown as in Figure 5 below.

$<<$ Figure 5. Line speed from VPN server to Malaysia

(Source: http://www.speedtest.net/)>>

Based on these 2 tests, the local speed maximum achievable speed is $843 \mathrm{~kb} / \mathrm{s}$ and the latency $28 \mathrm{~ms}$. The Jaring speed test overall speed included the international line from the origin of VPN to end user maximum achievable speed is $1085 \mathrm{~kb} / \mathrm{s}$ with a latency $314 \mathrm{~ms}$ speedtest.net. However, to be more realistic the line speed of the user taking into account various parameters either known or unknown or simply taking the worse case of 30\% degradation the expected maximum achievable speed should be around $774 \mathrm{~kb} / \mathrm{s}$ [5]. This figure seems relevant to the Jaring's figure of $843 \mathrm{~kb} / \mathrm{s}$.

\subsection{Benchmark files}

In the next observation and analysis a benchmark file for download should be identified. The main criterion for this file is that it should be readily available at anytime for $24 / 7$.

Apparently it is known that Linux Ubuntu OS is one of it and it is readily available and very popular. Its download activities are active among Linux community. Moreover it is also considered to have a stable download speed where it has enough number of seeds and peers. Thus this file is chosen to be the main benchmark download file. Two tests are conducted on this particular file. One will be downloading without VPN and the next with VPN deployed. With the test results this shall be the basis benchmark of the performance. The $24 / 7$ is required to gauge whether the time of day or the working days of a week affect the VPN download speed or otherwise.

\subsection{Ubuntu downloads without and with VPN}

For the initial observation on the Linux Ubuntu OS file it is downloaded using uTorrent in a normal way without VPN. The uTorrent client software is used due to it is currently the top BitTorrent software surpassing Azureus (http://torrentfreak.com/p2p-statistics-080426/). Figure 6 shows uTorrent in action downloading Ubuntu without VPN.

$<<$ Figure 6. uTorrent downloading ubuntu without VPN $>>$

Table 1 below shows the data gathered for a selected hour within 24/7 for analysis and Graph 1 shows the performance in a graphical view. Briefly it is noted that from the Table 1 below the performance of the uTorrent downloading speed on TMNET Streamyx is unstable and slow for 24/7 irrespective of hours of day or whether it is during the working days of the week or otherwise.

$<<$ Table 1. Ubuntu download performance without VPN for 24/7>>

$<<$ Graph 1. Ubuntu download speed without VPN for 24/7>>

Additionally the following facts are noted where the download speed ranges from as low as $2.8 \mathrm{kB} / \mathrm{s}$ and as high as $38.9 \mathrm{kB} / \mathrm{s}$. For $24 / 7$ the average download speed is $25 \mathrm{kB} / \mathrm{s}$. The number of seed only exist around 27 which means each seed only contribute less than $1 \mathrm{kB} / \mathrm{s}$. Although the download speed figure average at around $30 \mathrm{kB} / \mathrm{s}$, this file is accepted as a benchmark for testing because the file is available $24 / 7$. 
On the other hand Table 2 shows the results taken throughout 24/7 by downloading Ubuntu deploying VPN see also Figure 7 shows uTorrent in action downloading Ubuntu with VPN. The purpose of this process is to observe whether it is bounded by hours of day or the working hours of the week. Graph 2 shows the performance in graphical view. Briefly it is noted that from the Table 2 and Graph 2 as below the performance of the downloading speed is greatly improved. It is stable irrespective of hours of day or whether it is during the working day of the week. Thus in brief VPN has elevated the download process, improved instability and improved download speed. Hence this Ubuntu is correctly chosen as a benchmark file. It is important that the ISP connection line to the user and the number of seeds be considered. It is encouraged that the download speed ranges from as low as $97.4 \mathrm{kB} / \mathrm{s}$ and maximized at $117.2 \mathrm{kB} / \mathrm{s}$. For $24 / 7$ the average download speed is about over $110.6 \mathrm{kB} / \mathrm{s}$. The number of seed increases to around 70 which means each seed contributes more than $1 \mathrm{kB} / \mathrm{s}$.

$<<$ Figure 7. uTorrent downloading ubuntu with VPN $>>$

$<<$ Table 2. Ubuntu download performance with VPN for 24/7>>

$<<$ Graph 2. Ubuntu download speed with VPN for 24/7>>

\subsection{Random files downloaded without and with VPN}

The next observation is done on random files which basically end user will do. Two tests are conducted on this particular file. One will be downloading without VPN and the next with VPN deployed. Table 3 and Graph 3 below show the result for selected hours within 24/7 for analysis, see also Figure 8 below that shows uTorrent is in action downloading random files without VPN. Briefly it can be noted that from the Table 3 below the performance of the uTorrent downloading speed on TMNET Streamyx is unstable and slow for 24/7 irrespective of hours of day or whether it is during the working days of the week or otherwise.

It is to be noted that the download speed ranges from as low as $3.1 \mathrm{kB} / \mathrm{s}$ and maximized at $44.9 \mathrm{kB} / \mathrm{s}$. For $24 / 7$ the average download speed is: $17 \mathrm{kB} / \mathrm{s}$. The number of seed only exist around 38 which means each seed only contribute less than $1 \mathrm{kB} / \mathrm{s}$.

$<<$ Table 3. Random files download performance without VPN for 24/7 >>

$<<$ Graph 3. Random files download speed without VPN for 24/7>>

$<<$ Figure 8. uTorrent downloading random files without VPN $>>$

In the final observation a random file is downloaded with VPN where Figure 9 below shows uTorrent is in action downloading random files with VPN. The result is tabulated as in Table 4 with Graph 4 showing the performance in a graphical view format.

$<<$ Table 4. Random files download performance with VPN for 24/7>>

$<<$ Graph 4. Random files download speed with VPN for 24/7>>

$<<$ Figure 9. uTorrent downloading random files with VPN $>>$

Observing Graph 4 there are few discrepancies on the download performance. A repeat downloads on the particular time and days are conducted later. The Table 5 shows the new data on those particular instances. The new data is then modified to the former Table 4 which gives rise to Table 6 and the final graph is plotted as in Graph 5 .

$<<$ Table 5. Remedial observation on the specific days and time $>>$

$<<$ Table 6. The new modifies random files download performance with VPN for 24/7>>

$<<$ Graph 5. The new modified random files download with VPN for 24/7>>

Based on the new data gathered the discrepancies that occurred were merely predominate at that point of time. It is to be noted that the maximum speed of $120.8 \mathrm{kB} / \mathrm{s}$ with 34 seeders, the minimum speed is $98.1 \mathrm{kB} / \mathrm{s}$ with 25 seeders, the average speed is $110 \mathrm{kB} / \mathrm{s}$ and the minimum seeder to give adequate speed is above 20 .

\section{Results and discussion}

From the observations carried out thus far VPN has in fact improved and elevated the download speed of any files. The outcome is seen to be stable and the speed may reach the maximum allowable figure. There is no obvious constrain bounded to it either time of day or day of the week is observed.

In general, it was found that the speed of download can be maintained at above $100 \mathrm{kB} / \mathrm{s}$ or about $20 \%$ of the allowable user maximum speed (Cheshire, 1996). From the observation it is also noted that when the user is seeding to other peers and the tracker is down the download speed is affected. This probable phenomenon happens during Monday, Thursday, Friday, Saturday and Sunday during which the data was first observed for the random files download. This is only a random state which may not be repeated and only prominent at that instant. 
Ignoring the time when uTorrent is seeding the download speed is mainly at around $100 \mathrm{kB} / \mathrm{s}$ or average at $110.4 \mathrm{kB} / \mathrm{s}$. However, if any discrepancy is considered the average download speed is nearly about $104.7 \mathrm{kB} / \mathrm{s}$ which is still above $100 \mathrm{kB} / \mathrm{s}$ average irrespective of times, days or weeks. On the other hand, when the download speed dips there are a few reasons to this phenomenon. It might be caused by the tracker which was downed at that time that resulted in the user uTorrent seeding to other peers or because the ISP line was bad. The number of seeds of around 30+ is enough to give the maximum download speed of $100 \mathrm{kB} / \mathrm{s}$ but the number of peers is again not that relevant.

From the result obtained the behavior of any random file download is always high when VPN is implemented. However to achieve the speed as high as the benchmark speed it is noted that the number of seeders must be adequately large or the quality of seeder must be good and finally the ISP line condition should also be good. Observing the behavior of random file with and without VPN in graphical view (Graph 2, Graph 4 and Graph 5), it can be stated that the performance is obviously uplifted with VPN.

\section{Conclusion}

Briefly this paper has clearly illustrated that VPN is obviously able to circumvent the ISP (Streamyx) throttling in Malaysia. The most important point to note is that the VPN server should be located on the high bandwidth backbone before the ISP could impose the throttling practice. And only then the end user will benefit the maximum bandwidth speed provided by the ISP. The other advantages gained by end user using VPN are the encrypted traffic where nobody can sniff the traffic even ISP. It is able to be surfed anonymously where the actual IP address of origin was not revealed and to be as if the surfer is from the server site IP. In addition, it is able to bypass site which has been blocked by the local ISP since the origin of the server is located elsewhere away from it.

The above results of the experiment are achieved where the condition of the service is in a normal condition. But if the line is of a non-quality condition the effect is not fully realized. But as in the normal way the service provided by Streamyx to home user is usually found to be erratic, unstable and unpredictable.

\section{References}

Asohan, A. (2007). The Star. Throttling broadband access for all, Available online: http://thestar.com.my/news/story.asp?file=/2007/2/8/focus/16811744\&sec=focus, Downloaded: August 13, 2007.

Cheshire, $\mathrm{S}$ (1996). Latency and the quest for Interactivity, Available online: http://www.stuartcheshire.org/papers/LatencyQuest.ps, Downloaded: 31 March 2008.

Filesharing Report Shows Explosive Growth for uTorrent, Available online: http://torrentfreak.com/p2p-statistics-080426/, Downloaded: 5 March 2008.

Teoh, S (2006). The Star. TM Net: P2P traffic clogging broadband, Available online: http://star-techcentral.com/tech/story.asp?file=/2006/3/1/technology/13540730\&sec=technology, Downloaded: August 13, 2007.

TM News Release (2007). TM leads 17-member Consortium of Telcos to establish Asia-America Gateway (AAG) The first submarine cable system linking South East Asia directly to USA, Available online: http://www.tm.com.my/about_tm/newsroom/2007/070427.htm, Downloaded: Feb 14, 2008.

Zeropaid.com (2008). Azureus released date from BitTorrent Throttling Plugin, Available online: http://www.zeropaid.com/news/9428/Azureus+Releases+Data+from+BitTorrent+Throttling+Plugin, $\quad$ Downloaded: August 4, 2008.

Table 1. Ubuntu download performance without VPN for 24/7

\begin{tabular}{|c|c|c|c|c|c|c|c|c|c|c|c|c|}
\hline Tme Day & $8 \mathrm{am}$ & $10 \mathrm{am}$ & 12 noon & $2 \mathrm{pm}$ & $4 \mathrm{pm}$ & $6 \mathrm{pm}$ & $8 \mathrm{pm}$ & $10 \mathrm{pm}$ & $\begin{array}{c}12 \\
\text { midnight }\end{array}$ & $2 \mathrm{am}$ & $4 \mathrm{am}$ & $6 \mathrm{am}$ \\
\hline \multirow[t]{3}{*}{ Monday } & $23.8 \mathrm{kB} / \mathrm{s}$ & $29.3 \mathrm{kB} / \mathrm{s}$ & $18.2 \mathrm{kB} / \mathrm{s}$ & $15.7 \mathrm{kB} / \mathrm{s}$ & $17.5 \mathrm{kB} / \mathrm{s}$ & $21.6 \mathrm{kB} / \mathrm{s}$ & $17.1 \mathrm{kB} / \mathrm{s}$ & $17.7 \mathrm{kB} / \mathrm{s}$ & $23.8 \mathrm{kB} / \mathrm{s}$ & $29.0 \mathrm{kB} / \mathrm{s}$ & $15.6 \mathrm{kB} / \mathrm{s}$ & $26.4 \mathrm{kB} / \mathrm{s}$ \\
\hline & S 27(50) & S 28(47) & S 21(37) & S 30(35) & S 22(35) & S 34(39) & $\mathrm{S} 23(38)$ & S $35(50)$ & S 33(46) & S 19(54) & S 35(55) & S 17(37) \\
\hline & $\mathrm{P} 6(82)$ & $\mathrm{P} 7(152)$ & P 5(15) & P 4(13) & P 2(15) & P 3(20) & $\mathrm{P} 0(6)$ & P 1(15) & P 3(56) & P 3(110) & P 2(196) & P 1(4) \\
\hline \multirow{2}{*}{ Tuesday } & S 4(11) & S 19(28) & S $30(40)$ & S $18(48)$ & S 26(53) & S 30(86) & S 35(114) & S 36(114) & S 34(111) & S 38(116) & S 35(46) & S 36(46) \\
\hline & P 1(6) & P 2(8) & P 6(35) & $\mathrm{P} 0(7)$ & P 6(20) & P 4(18) & P 1(68) & P $1(68)$ & P 1(9) & $\mathrm{P} 0(10)$ & P 2(9) & P 2(12) \\
\hline \multirow[t]{2}{*}{ Wednesday } & $30.0 \mathrm{kB} / \mathrm{s}$ & $34.9 \mathrm{kB} / \mathrm{s}$ & $36.7 \mathrm{kB} / \mathrm{s}$ & $37.8 \mathrm{kB} / \mathrm{s}$ & $35.9 \mathrm{kB} / \mathrm{s}$ & $34.1 \mathrm{kB} / \mathrm{s}$ & $36.2 \mathrm{kB} / \mathrm{s}$ & $33.8 \mathrm{kB} / \mathrm{s}$ & $27.4 \mathrm{kB} / \mathrm{s}$ & $37.0 \mathrm{kB} / \mathrm{s}$ & $36.8 \mathrm{kB} / \mathrm{s}$ & $33.8 \mathrm{kB} / \mathrm{s}$ \\
\hline & S 35(114) & S 32(124) & S 34(45) & S 29(37) & S $34(40)$ & S $32(40)$ & $\mathrm{S} 34(50)$ & S 32(37) & S 32(113) & S $30(121)$ & S 32(49) & S 34(41) \\
\hline
\end{tabular}




\begin{tabular}{|c|c|c|c|c|c|c|c|c|c|c|c|c|}
\hline \multirow{2}{*}{ Thursday } & $33.2 \mathrm{kB} / \mathrm{s}$ & $32.1 \mathrm{kB} / \mathrm{s}$ & $34.2 \mathrm{kB} / \mathrm{s}$ & $33.3 \mathrm{kB} / \mathrm{s}$ & $38.3 \mathrm{kB} / \mathrm{s}$ & $31.3 \mathrm{kB} / \mathrm{s}$ & $29.4 \mathrm{kB} / \mathrm{s}$ & $24.6 \mathrm{kB} / \mathrm{s}$ & $32.3 \mathrm{kB} / \mathrm{s}$ & $37.6 \mathrm{kB} / \mathrm{s}$ & $36.6 \mathrm{kB} / \mathrm{s}$ & $37.4 \mathrm{kB} / \mathrm{s}$ \\
\hline & S 30(37) & S 31(37) & S 29(38) & S 35(81) & $\mathrm{S} 34(50)$ & S 34(116) & S 27(94) & S 30(94) & S 32(75) & S 34(47) & S 34(115) & S 25(62) \\
\hline \multirow[t]{3}{*}{ Friday } & $16.1 \mathrm{kB} / \mathrm{s}$ & $16.7 \mathrm{kB} / \mathrm{s}$ & $15.7 \mathrm{kB} / \mathrm{s}$ & $15.5 \mathrm{kB} / \mathrm{s}$ & $14.4 \mathrm{kB} / \mathrm{s}$ & $25.1 \mathrm{kB} / \mathrm{s}$ & $22.3 \mathrm{kB} / \mathrm{s}$ & $25.7 \mathrm{kB} / \mathrm{s}$ & $25.4 \mathrm{kB} / \mathrm{s}$ & $30.6 \mathrm{kB} / \mathrm{s}$ & $22.4 \mathrm{kB} / \mathrm{s}$ & $26.0 \mathrm{kB} / \mathrm{s}$ \\
\hline & S 34(45) & S 34(52) & S 29(37) & S 31(119) & S $33(58)$ & S 29(40) & S 27(35) & S 24(34) & S $28(68)$ & S $31(70)$ & S 32(39) & S 27(34) \\
\hline & P 1(115) & P 3(112) & P $4(12)$ & P $5(15)$ & P 5(84) & P $3(17)$ & P 3(10) & P 2(8) & P 2(13) & P 3(18) & P $3(16)$ & P 3(9) \\
\hline \multirow[t]{3}{*}{ Saturday } & $25.8 \mathrm{kB} / \mathrm{s}$ & $14.7 \mathrm{kB} / \mathrm{s}$ & $27.7 \mathrm{kB} / \mathrm{s}$ & $23.2 \mathrm{kB} / \mathrm{s}$ & $2.8 \mathrm{kB} / \mathrm{s}$ & $7.3 \mathrm{kB} / \mathrm{s}$ & $20.7 \mathrm{kB} / \mathrm{s}$ & $16.8 \mathrm{kB} / \mathrm{s}$ & $28.3 \mathrm{kB} / \mathrm{s}$ & $33.1 \mathrm{kB} / \mathrm{s}$ & $29.2 \mathrm{kB} / \mathrm{s}$ & $38.9 \mathrm{kB} / \mathrm{s}$ \\
\hline & S 26(36) & S 32(41) & S 16(37) & S 30(33) & $\mathrm{S} 12(30)$ & S 30(39) & S 16(34) & S 28(34) & S 16(32) & S 18(33) & S 23(29) & S 26(30) \\
\hline & P $0(9)$ & P 5(33) & P 0(16) & P 4(6) & Р 0(9) & P $6(71)$ & P 2(19) & P $6(17)$ & P 4(67) & P 6(67) & P 2(65) & P 5(67) \\
\hline \multirow[t]{2}{*}{ Sunday } & $27.1 \mathrm{kB} / \mathrm{s}$ & $34.6 \mathrm{kB} / \mathrm{s}$ & $36.7 \mathrm{kB} / \mathrm{s}$ & $18.3 \mathrm{kB} / \mathrm{s}$ & $14.6 \mathrm{kB} / \mathrm{s}$ & $25.0 \mathrm{kB} / \mathrm{s}$ & $17.8 \mathrm{kB} / \mathrm{s}$ & $16.6 \mathrm{kB} / \mathrm{s}$ & $17.9 \mathrm{kB} / \mathrm{s}$ & $16.6 \mathrm{kB} / \mathrm{s}$ & $15.8 \mathrm{kB} / \mathrm{s}$ & $25.0 \mathrm{kB} / \mathrm{s}$ \\
\hline & P 3(117) & P 2(74) & P 2(166) & $\mathrm{P} 0(6)$ & P 4(11) & P 1(12) & P 2(122) & P 1(10) & P 3(10) & P 4(12) & P 8(17) & P 2(13) \\
\hline
\end{tabular}

Table 2. Ubuntu download performance with VPN for 24/7

\begin{tabular}{|c|c|c|c|c|c|c|c|c|c|c|c|c|}
\hline $\begin{array}{l}\text { Time } \\
\text { Day }\end{array}$ & $8 \mathrm{am}$ & $10 \mathrm{am}$ & $\begin{array}{c}12 \\
\text { noon }\end{array}$ & $2 \mathrm{pm}$ & $4 \mathrm{pm}$ & $6 \mathrm{pm}$ & $8 \mathrm{pm}$ & $10 \mathrm{pm}$ & $\begin{array}{l}12 \\
\text { midnight }\end{array}$ & $2 \mathrm{am}$ & $4 \mathrm{am}$ & $6 \mathrm{am}$ \\
\hline \multirow[t]{3}{*}{ Monday } & $108.3 \mathrm{kB} / \mathrm{s}$ & $112.4 \mathrm{kB} / \mathrm{s}$ & $110.1 \mathrm{kB} / \mathrm{s}$ & $110.6 \mathrm{kB} / \mathrm{s}$ & $103.1 \mathrm{kB} / \mathrm{s}$ & $105.1 \mathrm{kB} / \mathrm{s}$ & $112.6 \mathrm{kB} / \mathrm{s}$ & $111.4 \mathrm{kB} / \mathrm{s}$ & $105.4 \mathrm{kB} / \mathrm{s}$ & $106.6 \mathrm{kB} / \mathrm{s}$ & $104.6 \mathrm{kB} / \mathrm{s}$ & $105.5 \mathrm{kB} / \mathrm{s}$ \\
\hline & S 72(1252) & S 68(1256) & S 69(1260) & S 56(1288) & S 29(1235) & S 67(1246) & S 71(1261) & S 69(1265) & S 69(1246) & S 67(1261) & S 70(1261) & S 70(1279) \\
\hline & P 3(72) & P 5(71) & P 6(75) & P 2(90) & P 2(99) & P $5(100)$ & P 4(76) & P 6(76) & P 1(100) & P 4(102) & P 3(102) & P 2(103) \\
\hline \multirow[t]{3}{*}{ Tuesday } & $112.1 \mathrm{kB} / \mathrm{s}$ & 104.1 & $114.3 \mathrm{kB} / \mathrm{s}$ & $111.0 \mathrm{kB} / \mathrm{s}$ & $111.1 \mathrm{kB} / \mathrm{s}$ & $111.2 \mathrm{kB} / \mathrm{s}$ & $109.7 \mathrm{kB} / \mathrm{s}$ & $110.8 \mathrm{kB} / \mathrm{s}$ & $112.3 \mathrm{kB} / \mathrm{s}$ & $112.9 \mathrm{kB} / \mathrm{s}$ & $113.0 \mathrm{kB} / \mathrm{s}$ & $113.6 \mathrm{kB} / \mathrm{s}$ \\
\hline & S 68(1197) & S 69(1204) & S 72(1267) & S 35(1207) & S 73(1207) & S 75(1213) & S 36(380) & S 71(1272) & S 73(1213) & S 74(1227) & S 74(1235) & S 74(1241) \\
\hline & P 6(92) & P 6(88) & P 3(77) & P $0(89)$ & P 2(89) & P $1(88)$ & P 1(22) & $\mathrm{P} 4(80)$ & P 2(88) & P $1(89)$ & P $1(87)$ & P $1(89)$ \\
\hline \multirow[t]{3}{*}{ Wednesday } & $110.3 \mathrm{kB} / \mathrm{s}$ & $113.6 \mathrm{kB} / \mathrm{s}$ & $115.2 \mathrm{kB} / \mathrm{s}$ & $111.3 \mathrm{kB} / \mathrm{s}$ & $111.8 \mathrm{kB} / \mathrm{s}$ & $106.4 \mathrm{kB} / \mathrm{s}$ & $111.8 \mathrm{kB} / \mathrm{s}$ & $111.9 \mathrm{kB} / \mathrm{s}$ & $109.4 \mathrm{kB} / \mathrm{s}$ & $108.8 \mathrm{kB} / \mathrm{s}$ & $117.2 \mathrm{kB} / \mathrm{s}$ & $112.1 \mathrm{kB} / \mathrm{s}$ \\
\hline & S 72(1203) & S 66(1218) & S 73(1229) & S 74(1235) & S 73(1240) & S 61(1242) & S 68(1247) & S 72(1251) & S 60(1242) & S 73(1242) & S 74(1205) & S 74(1205) \\
\hline & P 4(74) & P 3(77) & P 2(74) & $\mathrm{P} 0(76)$ & P 2(75) & P $3(78)$ & P 2(77) & P 2(77) & $\mathrm{P} 2(78)$ & P 2(78) & P 2(69) & P 2(69) \\
\hline \multirow[t]{3}{*}{ Thursday } & $110.4 \mathrm{kB} / \mathrm{s}$ & $105.3 \mathrm{kB} / \mathrm{s}$ & $111.8 \mathrm{kB} / \mathrm{s}$ & $109.2 \mathrm{kB} / \mathrm{s}$ & $112.1 \mathrm{kB} / \mathrm{s}$ & $111.4 \mathrm{kB} / \mathrm{s}$ & $109.4 \mathrm{kB} / \mathrm{s}$ & $112.8 \mathrm{kB} / \mathrm{s}$ & $109.7 \mathrm{kB} / \mathrm{s}$ & $112.2 \mathrm{kB} / \mathrm{s}$ & $114.2 \mathrm{kB} / \mathrm{s}$ & $111.3 \mathrm{kB} / \mathrm{s}$ \\
\hline & S 72(1249) & S 70(1214) & S 73(1258) & S 72(1267) & S 72(1267) & S 73(1211) & $\mathrm{S} 47(62)$ & S 71(265) & S 73(238) & S 71(132) & $\mathrm{S} 73(636)$ & S 73(774) \\
\hline & P 3(79) & P 3(82) & $\mathrm{P} 2(82)$ & $\mathrm{P} 2(81)$ & P 2(82) & P 1(73) & P 5(107) & P 4(236) & P 3(171) & P 3(398) & P 2(33) & P 2(41) \\
\hline \multirow[t]{3}{*}{ Friday } & $110.9 \mathrm{kB} / \mathrm{s}$ & $108.8 \mathrm{kB} / \mathrm{s}$ & $111.2 \mathrm{kB} / \mathrm{s}$ & $106 \mathrm{kB} / \mathrm{s}$ & $111.8 \mathrm{kB} / \mathrm{s}$ & $114.7 \mathrm{kB} / \mathrm{s}$ & $107.5 \mathrm{kB} / \mathrm{s}$ & $111.5 \mathrm{kB} / \mathrm{s}$ & $110.9 \mathrm{kB} / \mathrm{s}$ & $114.5 \mathrm{kB} / \mathrm{s}$ & $113.0 \mathrm{kB} / \mathrm{s}$ & $112.5 \mathrm{kB} / \mathrm{s}$ \\
\hline & S 75(499) & S 66(1262) & S 73(612) & S 74(963) & S 75(723) & S 74(810) & S 73(963) & S 75(885) & S 71(963) & S 71(1170) & S 73(1182) & S 47(1185) \\
\hline & P $0(32)$ & P 5(87) & P 1(37) & $\mathrm{P} 2(51)$ & $\mathrm{P} 0(42)$ & $\mathrm{P} 0(47)$ & P 1(51) & $\mathrm{P} 0(52)$ & P 1(51) & P 1(59) & P 2(60) & P 1(61) \\
\hline \multirow[t]{3}{*}{ Saturday } & $111.1 \mathrm{kB} / \mathrm{s}$ & $113.2 \mathrm{kB} / \mathrm{s}$ & $112.4 \mathrm{kB} / \mathrm{s}$ & $112.1 \mathrm{kB} / \mathrm{s}$ & $111.2 \mathrm{kB} / \mathrm{s}$ & $109.7 \mathrm{kB} / \mathrm{s}$ & $110.1 \mathrm{kB} / \mathrm{s}$ & $111.5 \mathrm{kB} / \mathrm{s}$ & $111.0 \mathrm{kB} / \mathrm{s}$ & $111.2 \mathrm{k} \mid \mathrm{B} / \mathrm{s}$ & $110.0 \mathrm{kB} / \mathrm{s}$ & $111.4 \mathrm{kB} / \mathrm{s}$ \\
\hline & S 75(1038) & S 74(1114) & S 75(1163) & S 76(1180) & S 74(1184) & S 71(1181) & S 72(1188) & S 73(1196) & S 74(1192) & S 73(1192) & S 73(1194) & S 74(1201) \\
\hline & P 1(68) & P 0(71) & P 0(72) & $\mathrm{P} 0(72)$ & P 1(74) & P 3(73) & P 3(73) & P 2(74) & P 2(66) & P 2(66) & P 2(66) & P 1(66) \\
\hline \multirow[t]{3}{*}{ Sunday } & $113.1 \mathrm{kB} / \mathrm{s}$ & $111.5 \mathrm{kB} / \mathrm{s}$ & $112.6 \mathrm{kB} / \mathrm{s}$ & $111.2 \mathrm{kB} / \mathrm{s}$ & $97.4 \mathrm{kB} / \mathrm{s}$ & $110.8 \mathrm{kB} / \mathrm{s}$ & $111.9 \mathrm{kB} / \mathrm{s}$ & $109.3 \mathrm{kB} / \mathrm{s}$ & $112.3 \mathrm{kB} / \mathrm{s}$ & $112.2 \mathrm{kB} / \mathrm{s}$ & $109.0 \mathrm{kB} / \mathrm{s}$ & $113.2 \mathrm{kB} / \mathrm{s}$ \\
\hline & S 74(1214) & S $72(1230)$ & S 73(1239) & S 72(1245) & S 52(1342) & S 77(1204) & S 73(1244) & S 75(1256) & S 24(1204) & $\mathrm{S} 72(1222)$ & $\mathrm{S} 71(1225)$ & S 74(1230) \\
\hline & P 1(78) & P 2(80) & P 2(81) & P 2(81) & P $5(79)$ & P 1(67) & P 1(82) & P 1(82) & P $0(67)$ & P 3(67) & P 3(68) & P 1(71) \\
\hline
\end{tabular}

\section{Note:}

1. The download speed is in $\mathrm{kByte} / \mathrm{s}$

2. $\mathrm{Sn} 1(\mathrm{n} 2)$ where $\mathrm{n} 1$ is the number of seed which is currently connected, while $\mathrm{n} 2$ is the number of seed which is active globally

3. $\mathrm{P} n 1(\mathrm{n} 2)$ where $\mathrm{n} 1$ is the number of peer which is currently connected, while $\mathrm{n} 2$ is the number of peer which is active globally 
Table 3. Random files download performance without VPN for 24/7

\begin{tabular}{|c|c|c|c|c|c|c|c|c|c|c|c|c|}
\hline Day & $8 \mathrm{am}$ & $10 \mathrm{am}$ & $\begin{array}{c}12 \\
\text { noon }\end{array}$ & $2 \mathrm{pm}$ & $4 \mathrm{pm}$ & $6 \mathrm{pm}$ & $8 p m$ & 10 pm & $\begin{array}{c}12 \\
\text { midnight }\end{array}$ & $2 \mathrm{am}$ & $4 \mathrm{am}$ & $6 \mathrm{am}$ \\
\hline Monday & $\begin{array}{c}29.8 \mathrm{kB} / \mathrm{s} \\
\mathrm{S} 31(1134) \\
\text { P 1(125) }\end{array}$ & $\begin{array}{c}28.6 \mathrm{kB} / \mathrm{s} \\
\mathrm{S} 34(1138) \\
\text { P } 0(124)\end{array}$ & $\begin{array}{c}30.9 \mathrm{kB} / \mathrm{s} \\
\mathrm{S} 37(1138) \\
\text { P } 0(124)\end{array}$ & $\begin{array}{c}24.7 \mathrm{kB} / \mathrm{s} \\
\mathrm{S} 37(1138) \\
\text { P } 0(124)\end{array}$ & $\begin{array}{c}36.5 \mathrm{kB} / \mathrm{s} \\
\mathrm{S} 34(1138) \\
\text { P 2(121) }\end{array}$ & $\begin{array}{l}24.1 \mathrm{kB} / \mathrm{s} \\
\mathrm{S} 31(1164) \\
\text { P 2(114) }\end{array}$ & $\begin{array}{c}29.7 \mathrm{kB} / \mathrm{s} \\
\text { S } 34(1159) \\
\text { P } 2(114)\end{array}$ & $\begin{array}{c}34.7 \mathrm{kB} / \mathrm{s} \\
\mathrm{S} 33(1153) \\
\text { P 2(113) }\end{array}$ & $\begin{array}{l}10.6 \mathrm{kB} / \mathrm{s} \\
\text { S } 5(1156) \\
\text { P 0(113) }\end{array}$ & $\begin{array}{l}25.1 \mathrm{kB} / \mathrm{s} \\
\mathrm{S} 35(1156) \\
\mathrm{P} 1(113)\end{array}$ & $\begin{array}{c}27.6 \mathrm{kB} / \mathrm{s} \\
\mathrm{S} 37(1156) \\
\text { P } 0(113)\end{array}$ & $\begin{array}{c}23.1 \mathrm{kB} / \mathrm{s} \\
\mathrm{S} 38(1156) \\
\text { P } 0(113)\end{array}$ \\
\hline Tuesday & $\begin{array}{c}25.9 \mathrm{kB} / \mathrm{s} \\
\mathrm{S} 34(1161) \\
\text { P 2(109) }\end{array}$ & $\begin{array}{c}15.1 \mathrm{kB} / \mathrm{s} \\
\mathrm{S} 35(1161) \\
\text { P } 2(109)\end{array}$ & $\begin{array}{c}26.1 \mathrm{kB} / \mathrm{s} \\
\mathrm{S} 36(1171) \\
\text { P } 1(108)\end{array}$ & $\begin{array}{c}3.1 \mathrm{kB} / \mathrm{s} \\
\mathrm{S} 12(1479) \\
\mathrm{P} 0(147)\end{array}$ & $\begin{array}{c}7.0 \mathrm{kB} / \mathrm{s} \\
\mathrm{S} 12(1479) \\
\text { P } 0(147)\end{array}$ & $\begin{array}{c}5.1 \mathrm{kB} / \mathrm{s} \\
\mathrm{S} 18(1490) \\
\text { P } 0(148)\end{array}$ & $\begin{array}{c}5.6 \mathrm{kB} / \mathrm{s} \\
\mathrm{S} 29(1482) \\
\text { P 1(147) }\end{array}$ & $\begin{array}{c}4.0 \mathrm{kB} / \mathrm{s} \\
\text { S } 24(1482) \\
\text { P } 0(147)\end{array}$ & $\begin{array}{c}14.9 \mathrm{kB} / \mathrm{s} \\
\mathrm{S} 25(1482) \\
\text { P } 1(147)\end{array}$ & $\begin{array}{c}7.5 \mathrm{kB} / \mathrm{s} \\
\mathrm{S} 29(1482) \\
\text { P } 1(147)\end{array}$ & $\begin{array}{c}3.9 \mathrm{kB} / \mathrm{s} \\
\mathrm{S} 28(1482) \\
\text { P } 0(147)\end{array}$ & $\begin{array}{c}4.3 \mathrm{kB} / \mathrm{s} \\
\mathrm{S} 23(1482) \\
\text { P } 0(147)\end{array}$ \\
\hline Wednesday & $\begin{array}{c}4.9 \mathrm{kB} / \mathrm{s} \\
\mathrm{S} 30(1482) \\
\text { P } 1(147)\end{array}$ & $\begin{array}{c}10.4 \mathrm{kB} / \mathrm{s} \\
\mathrm{S} 28(1468) \\
\text { P } 0(136)\end{array}$ & $\begin{array}{c}13.7 \mathrm{kB} / \mathrm{s} \\
\mathrm{S} 22(1468) \\
\mathrm{P} 0(136)\end{array}$ & $\begin{array}{c}7.5 \mathrm{kB} / \mathrm{s} \\
\mathrm{S} 29(1476) \\
\mathrm{P} 0(136)\end{array}$ & $\begin{array}{c}20.2 \mathrm{kB} / \mathrm{s} \\
\mathrm{S} 33(1476) \\
\text { P } 0(136)\end{array}$ & $\begin{array}{c}24.0 \mathrm{kB} / \mathrm{s} \\
\mathrm{S} 32(1476) \\
\text { P } 0(136)\end{array}$ & $\begin{array}{c}26.7 \mathrm{kB} / \mathrm{s} \\
\mathrm{S} 29(1476) \\
\text { P } 0(136)\end{array}$ & $\begin{array}{c}35.6 \mathrm{kB} / \mathrm{s} \\
\mathrm{S} 33(1476) \\
\text { P } 0(136)\end{array}$ & $\begin{array}{l}44.9 \mathrm{kB} / \mathrm{s} \\
\text { S } 27(1483) \\
\text { P } 0(120)\end{array}$ & $\begin{array}{l}33.9 \mathrm{kB} / \mathrm{s} \\
\mathrm{S} 28(1483) \\
\text { P } 0(120)\end{array}$ & $\begin{array}{l}12.3 \mathrm{kB} / \mathrm{s} \\
\mathrm{S} 13(1828) \\
\text { P 2(162) }\end{array}$ & $\begin{array}{c}4.4 \mathrm{kB} / \mathrm{s} \\
\mathrm{S} 11(1824) \\
\text { P } 0(165)\end{array}$ \\
\hline Thursday & $\begin{array}{c}4.4 \mathrm{kB} / \mathrm{s} \\
\mathrm{S} 26(1831) \\
\text { P } 2(167)\end{array}$ & $\begin{array}{c}14.1 \mathrm{kB} / \mathrm{s} \\
\mathrm{S} 36(1831) \\
\text { P } 0(167)\end{array}$ & $\begin{array}{c}11.6 \mathrm{kB} / \mathrm{s} \\
\mathrm{S} 30(1831) \\
\text { P } 0(167)\end{array}$ & $\begin{array}{c}7.8 \mathrm{kB} / \mathrm{s} \\
\mathrm{S} 30(1831) \\
\text { P } 0(167)\end{array}$ & $\begin{array}{c}12.7 \mathrm{kB} / \mathrm{s} \\
\mathrm{S} 23(1831) \\
\text { P } 0(167)\end{array}$ & $\begin{array}{c}25.7 \mathrm{kB} / \mathrm{s} \\
\mathrm{S} 33(1846) \\
\text { P } 1(170)\end{array}$ & $\begin{array}{c}15.7 \mathrm{kB} / \mathrm{s} \\
\mathrm{S} 26(1846) \\
\text { P } 1(170)\end{array}$ & $\begin{array}{c}18.8 \mathrm{kB} / \mathrm{s} \\
\mathrm{S} 25(1853) \\
\text { P } 3(172)\end{array}$ & $\begin{array}{c}9.6 \mathrm{kB} / \mathrm{s} \\
\mathrm{S} 27(1846) \\
\text { P } 1(176)\end{array}$ & $\begin{array}{c}12.9 \mathrm{kB} / \mathrm{s} \\
\mathrm{S} 32(1846) \\
\mathrm{P} 0(176)\end{array}$ & $\begin{array}{c}11.4 \mathrm{kB} / \mathrm{s} \\
\mathrm{S} 18(1846) \\
\text { P } 0(176)\end{array}$ & $\begin{array}{c}26.7 \mathrm{kB} / \mathrm{s} \\
\mathrm{S} 30(1846) \\
\text { P } 0(176)\end{array}$ \\
\hline Friday & $\begin{array}{c}7.0 \mathrm{kB} / \mathrm{s} \\
\mathrm{S} 12(1738) \\
\text { P } 0(137)\end{array}$ & $\begin{array}{c}7.4 \mathrm{kB} / \mathrm{s} \\
\mathrm{S} 24(1738) \\
\text { P } 1(137)\end{array}$ & $\begin{array}{c}9.5 \mathrm{kB} / \mathrm{s} \\
\mathrm{S} 23(1738) \\
\text { P } 2(137)\end{array}$ & $\begin{array}{c}8.6 \mathrm{kB} / \mathrm{s} \\
\mathrm{S} 25(1728) \\
\text { P } 2(144)\end{array}$ & $\begin{array}{c}12 \mathrm{kB} / \mathrm{s} \\
\mathrm{S} 22(1728) \\
\mathrm{P} 2(144)\end{array}$ & $\begin{array}{c}13.3 \mathrm{kB} / \mathrm{s} \\
\text { S } 23(1737) \\
\text { P } 3(143)\end{array}$ & $\begin{array}{c}16.9 \mathrm{kB} / \mathrm{s} \\
\mathrm{S} 30(1737) \\
\text { P } 3(143)\end{array}$ & $\begin{array}{c}7.1 \mathrm{kB} / \mathrm{s} \\
\text { S 28(1737) } \\
\text { P 5(143) }\end{array}$ & $\begin{array}{c}9.1 \mathrm{kB} / \mathrm{s} \\
\text { S } 32(1737) \\
\text { P 2(143) }\end{array}$ & $\begin{array}{c}19.0 \mathrm{kB} / \mathrm{s} \\
\mathrm{S} 21(1737) \\
\mathrm{P} 0(143)\end{array}$ & $\begin{array}{c}20.9 \mathrm{kB} / \mathrm{s} \\
\mathrm{S} 25(1737) \\
\text { P } 0(143)\end{array}$ & $\begin{array}{c}21.0 \mathrm{kB} / \mathrm{s} \\
\text { S 16(1737) } \\
\text { P 0(143) }\end{array}$ \\
\hline Saturday & $\begin{array}{c}9.6 \mathrm{kB} / \mathrm{s} \\
\mathrm{S} 14(1737) \\
\text { P } 0(117)\end{array}$ & $\begin{array}{c}8.9 \mathrm{kB} / \mathrm{s} \\
\mathrm{S} 24(1737) \\
\text { P } 1(117)\end{array}$ & $\begin{array}{c}22.5 \mathrm{kB} / \mathrm{s} \\
\mathrm{S} 28(1737) \\
\text { P } 1(117)\end{array}$ & $\begin{array}{c}16.6 \mathrm{kB} / \mathrm{s} \\
\mathrm{S} 28(1737) \\
\text { P } 0(117)\end{array}$ & $\begin{array}{c}16.4 \mathrm{kB} / \mathrm{s} \\
\mathrm{S} 27(1737) \\
\text { P } 1(117)\end{array}$ & $\begin{array}{c}12.6 \mathrm{kB} / \mathrm{s} \\
\mathrm{S} 25(1737) \\
\text { P } 0(117)\end{array}$ & $\begin{array}{l}15.2 \mathrm{kB} / \mathrm{s} \\
\mathrm{S} 2(1725) \\
\mathrm{P} 0(124)\end{array}$ & $\begin{array}{c}4.9 \mathrm{kB} / \mathrm{s} \\
\mathrm{S} 13(1723) \\
\text { P } 0(124)\end{array}$ & $\begin{array}{c}12.7 \mathrm{kB} / \mathrm{s} \\
\mathrm{S} 24(1720) \\
\text { P } 1(125)\end{array}$ & $\begin{array}{c}16.9 \mathrm{kB} / \mathrm{s} \\
\mathrm{S} 14(1720) \\
\mathrm{P} 0(125)\end{array}$ & $\begin{array}{c}33.9 \mathrm{kB} / \mathrm{s} \\
\mathrm{S} 26(1720) \\
\text { P } 1(125)\end{array}$ & $\begin{array}{c}27.1 \mathrm{kB} / \mathrm{s} \\
\mathrm{S} 26(1720) \\
\text { P } 1(125)\end{array}$ \\
\hline Sunday & $\begin{array}{l}4.7 \mathrm{kB} / \mathrm{s} \\
\text { S } 4(1129) \\
\text { P } 1(127)\end{array}$ & $\begin{array}{l}4.0 \mathrm{kB} / \mathrm{s} \\
\text { S } 3(1129) \\
\text { P } 1(127)\end{array}$ & $\begin{array}{c}6.7 \mathrm{kB} / \mathrm{s} \\
\mathrm{S} 7(1137) \\
\text { P } 1(129)\end{array}$ & $\begin{array}{c}9.2 \mathrm{kB} / \mathrm{s} \\
\mathrm{S} 10(1141) \\
\text { P } 3(129)\end{array}$ & $\begin{array}{l}13.0 \mathrm{kB} / \mathrm{s} \\
\mathrm{S} 15(1141) \\
\text { P } 3(129)\end{array}$ & $\begin{array}{c}21.1 \mathrm{kB} / \mathrm{s} \\
\mathrm{S} 23(1142) \\
\text { P } 4(127)\end{array}$ & $\begin{array}{c}18.2 \mathrm{kB} / \mathrm{s} \\
\mathrm{S} 22(1135) \\
\text { P } 5(128)\end{array}$ & $\begin{array}{l}31.6 \mathrm{kB} / \mathrm{s} \\
\mathrm{S} 32(1132) \\
\text { P } 4(126)\end{array}$ & $\begin{array}{l}11.8 \mathrm{kB} / \mathrm{s} \\
\text { S } 3(1134) \\
\text { P } 0(125)\end{array}$ & $\begin{array}{c}16.6 \mathrm{kB} / \mathrm{s} \\
\mathrm{S} 32(1134) \\
\text { P } 0(125)\end{array}$ & $\begin{array}{c}24.3 \mathrm{kB} / \mathrm{s} \\
\mathrm{S} 34(1134) \\
\text { P } 0(125)\end{array}$ & $\begin{array}{c}27.0 \mathrm{kB} / \mathrm{s} \\
\mathrm{S} 35(1134) \\
\text { P } 0(125)\end{array}$ \\
\hline
\end{tabular}

Table 4. Random files download performance with VPN for 24/7

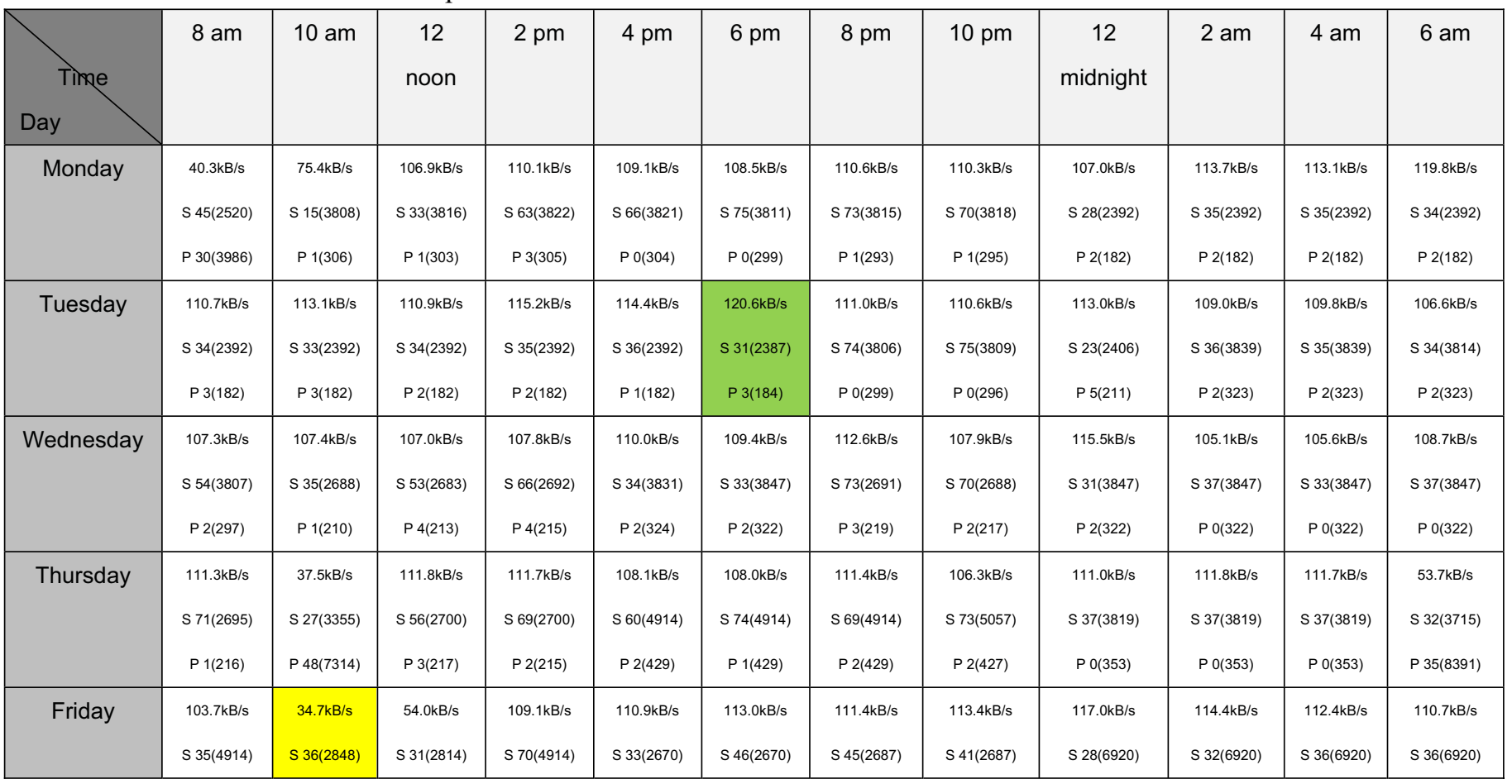


Vol. 2, No. 1

Computer and Information Science

\begin{tabular}{|c|c|c|c|c|c|c|c|c|c|c|c|c|}
\hline & P 5(429) & P 34(6282) & P 44(6204) & P 4(429) & P 3(239) & P 1(238) & $\mathrm{P} 0(240)$ & P $0(240)$ & P 1(711) & P 1(711) & P 1(711) & P 1(711) \\
\hline \multirow[t]{3}{*}{ Saturday } & $109.7 \mathrm{kB} / \mathrm{s}$ & $111.2 \mathrm{kB} / \mathrm{s}$ & $109.6 \mathrm{kB} / \mathrm{s}$ & $108.5 \mathrm{kB} / \mathrm{s}$ & $109.3 \mathrm{kB} / \mathrm{s}$ & $111.0 \mathrm{kB} / \mathrm{s}$ & $107.8 \mathrm{kB} / \mathrm{s}$ & $108.0 \mathrm{kB} / \mathrm{s}$ & $108.4 \mathrm{kB} / \mathrm{s}$ & $67.4 \mathrm{kB} / \mathrm{s}$ & $108.8 \mathrm{kB} / \mathrm{s}$ & $109.1 \mathrm{kB} / \mathrm{s}$ \\
\hline & S 49(3822) & S 47(3822) & S 48(3853) & S 73(3875) & S 60(3876) & S 72(3886) & S 71(3874) & S 67(3888) & S 33(6915) & S 30(2721) & S 35(6915) & S 31(6915) \\
\hline & P 2(313) & P 1(313) & P 2(313) & P 2(309) & P 1(306) & P 0(307) & P 2(306) & P 1(301) & P 2(720) & P 44(5374) & P 2(720) & P 2(720) \\
\hline \multirow[t]{3}{*}{ Sunday } & $106.1 \mathrm{kB} / \mathrm{s}$ & $109.0 \mathrm{kB} / \mathrm{s}$ & $112.5 \mathrm{kB} / \mathrm{s}$ & $112.5 \mathrm{kB} / \mathrm{s}$ & $35.2 \mathrm{kB} / \mathrm{s}$ & $109.5 \mathrm{kB} / \mathrm{s}$ & $109.8 \mathrm{kB} / \mathrm{s}$ & $108.0 \mathrm{kB} / \mathrm{s}$ & $111.7 \mathrm{kB} / \mathrm{s}$ & $110.6 \mathrm{kB} / \mathrm{s}$ & $111.1 \mathrm{kB} / \mathrm{s}$ & $113.7 \mathrm{kB} / \mathrm{s}$ \\
\hline & S 73(3898) & S 73(3907) & S 27(2850) & S 29(2873) & S 38(3002) & S 37(6925) & S 35(6927) & S 38(6927) & S 37(6935) & S 37(6935) & S 36(6935) & S 37(6935) \\
\hline & P 1(299) & P 2(294) & P 0(211) & P 1(203) & P 26(5109) & P 0(712) & P 0(707) & P 0(707) & P 0(704) & P 0(704) & P 0(704) & P 0(704) \\
\hline
\end{tabular}

\section{Note:}

1. The download speed is in $\mathrm{kByte} / \mathrm{s}$

2. $\mathrm{Sn} 1(\mathrm{n} 2)$ where $\mathrm{n} 1$ is the number of seed which is currently connected, while $\mathrm{n} 2$ is the number of seed which is active globally

3. $\mathrm{P} n 1(\mathrm{n} 2)$ where $\mathrm{n} 1$ is the number of peer which is currently connected, while $\mathrm{n} 2$ is the number of peer which is active globally

Table 5. Remedial observation on the specific days and time

\begin{tabular}{|c|c|c|c|}
\hline Day & & Time & \\
\hline Monday & $\begin{array}{c}8 \mathrm{am} \\
98.1 \mathrm{kB} / \mathrm{s} \\
\mathrm{S} 25(6092) / \mathrm{P} 0(679)\end{array}$ & $\begin{array}{c}10 \mathrm{am} \\
114.2 \mathrm{kB} / \mathrm{s} \\
\mathrm{S} 36(6092) / \mathrm{P} 0(679)\end{array}$ & $\begin{array}{c}12 \mathrm{pm} \\
112.2 \mathrm{kB} / \mathrm{s} \\
\mathrm{S} 36(6092) / \mathrm{P} 0(679)\end{array}$ \\
\hline Thursday & $\begin{array}{c}8 \mathrm{am} \\
110.0 \mathrm{kB} / \mathrm{s} \\
\mathrm{S} \mathrm{32(6092)} / \mathrm{P} 0(679)\end{array}$ & $\begin{array}{c}10 \mathrm{am} \\
105.1 \mathrm{kB} / \mathrm{s} \\
\mathrm{S} 36(6092) / \mathrm{P} 1(679)\end{array}$ & $\begin{array}{c}6 \mathrm{am} \\
120.8 \mathrm{kB} / \mathrm{s} \\
\mathrm{S} 34(2419) / \mathrm{P} 1(243)\end{array}$ \\
\hline Friday & $\begin{array}{c}10 \mathrm{am} \\
108.3 \mathrm{kB} / \mathrm{s} \\
\mathrm{S} 24(6092) / \mathrm{P} 1(679)\end{array}$ & $\begin{array}{c}12 \mathrm{pm} \\
104.9 \mathrm{kB} / \mathrm{s} \\
\mathrm{S} 36(2419) / \mathrm{P} 1(243)\end{array}$ & $\begin{array}{c}2 \mathrm{pm} \\
107.8 \mathrm{kB} / \mathrm{s} \\
\mathrm{S} 36(6092) / \mathrm{P} 1(679)\end{array}$ \\
\hline Saturday & $\begin{array}{c}12 \mathrm{am} \\
108.7 \mathrm{kB} / \mathrm{s} \\
\mathrm{S} 36(6092) / \mathrm{P} 1(679)\end{array}$ & $\begin{array}{c}2 \mathrm{am} \\
108.5 \mathrm{kB} / \mathrm{s} \\
\mathrm{S} 36(6092) / \mathrm{P} 1(679)\end{array}$ & $\begin{array}{c}4 \mathrm{am} \\
111.3 \mathrm{kB} / \mathrm{s} \\
\mathrm{S} 36(6092) / \mathrm{P} 1(679)\end{array}$ \\
\hline Sunday & $\begin{array}{c}2 \mathrm{pm} \\
104.1 \mathrm{kB} / \mathrm{s} \\
\mathrm{S} 36(6092) / \mathrm{P} 1(679)\end{array}$ & $\begin{array}{c}4 \mathrm{pm} \\
111.0 \mathrm{kB} / \mathrm{s} \\
\mathrm{S} 36(6092) / \mathrm{P} 1(679)\end{array}$ & $\begin{array}{c}6 \mathrm{pm} \\
108.7 \mathrm{kB} / \mathrm{s} \\
\mathrm{S} 35(6092) / \mathrm{P} 1(679)\end{array}$ \\
\hline
\end{tabular}

Table 6. The new modifies random files download performance with VPN for 24/7

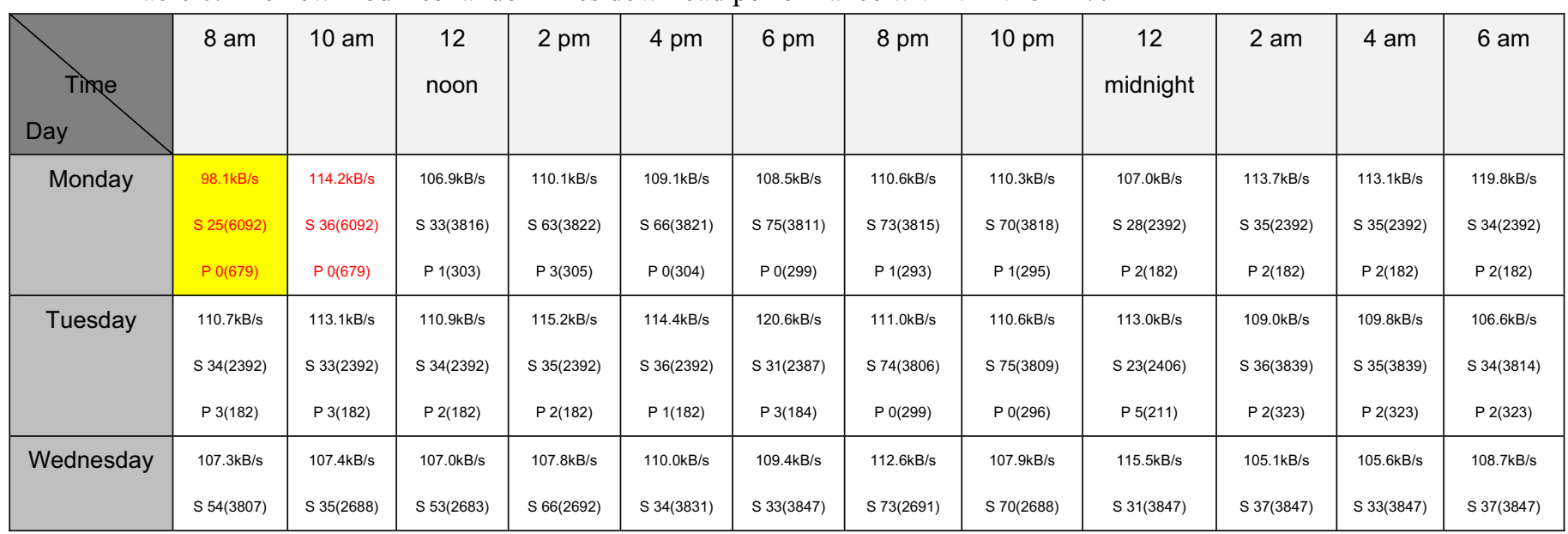




\begin{tabular}{|c|c|c|c|c|c|c|c|c|c|c|c|c|}
\hline & P 2(297) & P 1(210) & P 4(213) & P 4(215) & P 2(324) & P 2(322) & P 3(219) & P 2(217) & P 2(322) & P 0(322) & $\mathrm{P} 0(322)$ & P 0(322) \\
\hline \multirow[t]{3}{*}{ Thursday } & $111.3 \mathrm{kB} / \mathrm{s}$ & $105.1 \mathrm{kB} / \mathrm{s}$ & $111.8 \mathrm{kB} / \mathrm{s}$ & $111.7 \mathrm{kB} / \mathrm{s}$ & $108.1 \mathrm{kB} / \mathrm{s}$ & $108.0 \mathrm{kB} / \mathrm{s}$ & $111.4 \mathrm{kB} / \mathrm{s}$ & $106.3 \mathrm{kB} / \mathrm{s}$ & $111.0 \mathrm{kB} / \mathrm{s}$ & $111.8 \mathrm{kB} / \mathrm{s}$ & $111.7 \mathrm{kB} / \mathrm{s}$ & $120.8 \mathrm{kB} / \mathrm{s}$ \\
\hline & S 71(2695) & S 36(6092) & S 56(2700) & S 69(2700) & S 60(4914) & S 74(4914) & S 69(4914) & S 73(5057) & S 37(3819) & S 37(3819) & S $37(3819)$ & $S 34(2419) P$ \\
\hline & P 1(216) & P 1(679) & P 3(217) & P 2(215) & P 2(429) & P 1(429) & P 2(429) & P 2(427) & P 0(353) & P 0(353) & P 0(353) & $1(243)$ \\
\hline \multirow[t]{3}{*}{ Friday } & $103.7 \mathrm{kB} / \mathrm{s}$ & $108.3 \mathrm{kB} / \mathrm{s}$ & $104.9 \mathrm{kB} / \mathrm{s}$ & $109.1 \mathrm{kB} / \mathrm{s}$ & $110.9 \mathrm{kB} / \mathrm{s}$ & $113.0 \mathrm{kB} / \mathrm{s}$ & $111.4 \mathrm{kB} / \mathrm{s}$ & $113.4 \mathrm{kB} / \mathrm{s}$ & $117.0 \mathrm{kB} / \mathrm{s}$ & $114.4 \mathrm{kB} / \mathrm{s}$ & $112.4 \mathrm{kB} / \mathrm{s}$ & $110.7 \mathrm{kB} / \mathrm{s}$ \\
\hline & S 35(4914) & S 24(6092) & S 36(2419) & S 70(4914) & S 33(2670) & S 46(2670) & S 45(2687) & S 41(2687) & S 28(6920) & S 32(6920) & S 36(6920) & S 36(6920) \\
\hline & P 5(429) & P 1(679) & P 1(243) & P 4(429) & P $3(239)$ & P 1(238) & $\mathrm{P} 0(240)$ & $\mathrm{P} 0(240)$ & P 1(711) & P 1(711) & P 1(711) & P 1(711) \\
\hline \multirow[t]{3}{*}{ Saturday } & $109.7 \mathrm{kB} / \mathrm{s}$ & $111.2 \mathrm{kB} / \mathrm{s}$ & $109.6 \mathrm{kB} / \mathrm{s}$ & $108.5 \mathrm{kB} / \mathrm{s}$ & $109.3 \mathrm{kB} / \mathrm{s}$ & $111.0 \mathrm{kB} / \mathrm{s}$ & $107.8 \mathrm{kB} / \mathrm{s}$ & $108.0 \mathrm{kB} / \mathrm{s}$ & $108.4 \mathrm{kB} / \mathrm{s}$ & $108.5 \mathrm{kB} / \mathrm{s}$ & $108.8 \mathrm{kB} / \mathrm{s}$ & $109.1 \mathrm{kB} / \mathrm{s}$ \\
\hline & S 49(3822) & S 47(3822) & S 48(3853) & S 73(3875) & S 60(3876) & S 72(3886) & S 71(3874) & S 67(3888) & S 33(6915) & S 36(6092) / & S 35(6915) & S 31(6915) \\
\hline & P 2(313) & P 1(313) & P 2(313) & P 2(309) & P 1(306) & P 0(307) & P 2(306) & P 1(301) & P 2(720) & P 1(679) & P 2(720) & P 2(720) \\
\hline \multirow[t]{3}{*}{ Sunday } & $106.1 \mathrm{kB} / \mathrm{s}$ & $109.0 \mathrm{kB} / \mathrm{s}$ & $112.5 \mathrm{kB} / \mathrm{s}$ & $112.5 \mathrm{kB} / \mathrm{s}$ & $111.0 \mathrm{kB} / \mathrm{s}$ & $109.5 \mathrm{kB} / \mathrm{s}$ & $109.8 \mathrm{kB} / \mathrm{s}$ & $108.0 \mathrm{kB} / \mathrm{s}$ & $111.7 \mathrm{kB} / \mathrm{s}$ & $110.6 \mathrm{kB} / \mathrm{s}$ & $111.1 \mathrm{kB} / \mathrm{s}$ & $113.7 \mathrm{kB} / \mathrm{s}$ \\
\hline & S 73(3898) & S 73(3907) & S 27(2850) & S 29(2873) & S 36(6092) & S 37(6925) & S 35(6927) & S 38(6927) & S 37(6935) & S 37(6935) & S 36(6935) & S 37(6935) \\
\hline & P 1(299) & P 2(294) & P 0(211) & P 1(203) & / P 1(679) & P 0(712) & P 0(707) & P 0(707) & P 0(704) & P 0(704) & P 0(704) & P 0(704) \\
\hline
\end{tabular}

Note:

1. The download speed is in $\mathrm{kByte} / \mathrm{s}$

2. $\mathrm{Sn} 1(\mathrm{n} 2)$ where $\mathrm{n} 1$ is the number of seed which is currently connected, while $\mathrm{n} 2$ is the number of seed which is active globally

3. $\mathrm{P} n 1(\mathrm{n} 2)$ where $\mathrm{n} 1$ is the number of peer which is currently connected, while $\mathrm{n} 2$ is the number of peer which is active globally

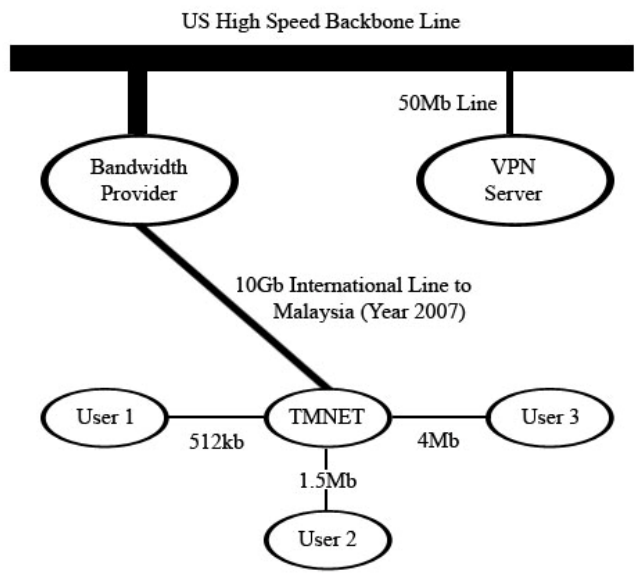

Figure 1. Location of VPN server

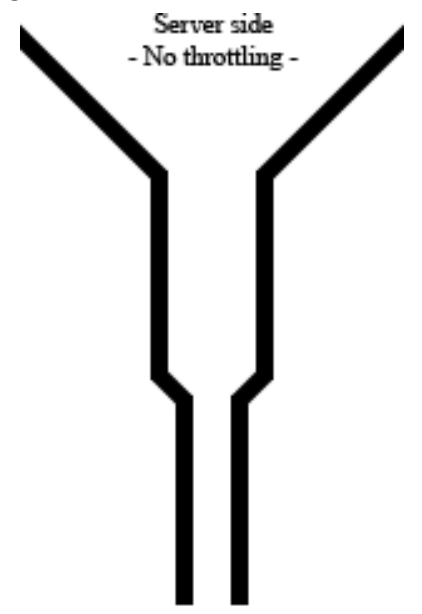

Client Side

- ISP bandwidth -

Figure 2 . The arbitrary tunnel created by VPN 


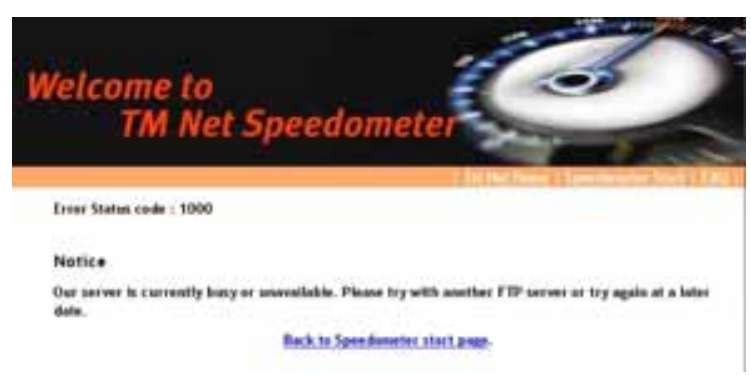

Figure 3. Streamyx speedometer speed test

(Source: http://202.188.95.52:8080/speedometer/)

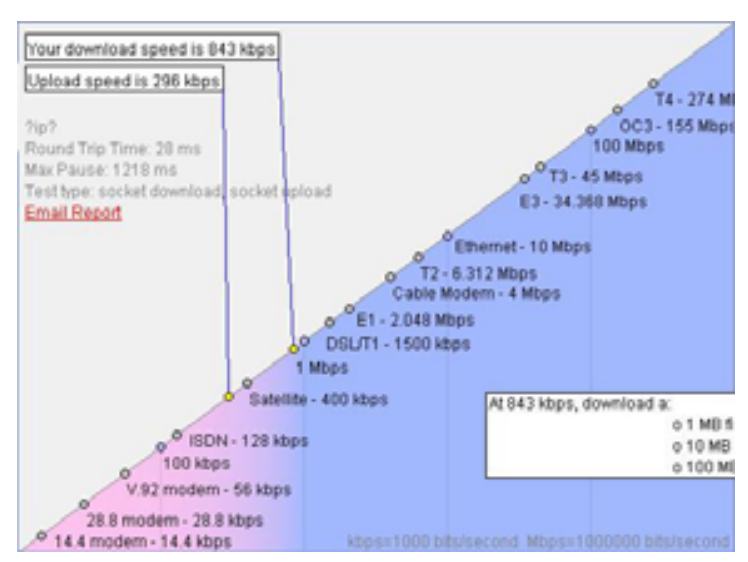

Figure 4. Speed test result given by Jaring ISP on Streamyx line.

(Source: http://www.jaring.my/service/broadband/index.cfm?cont=bandwidth_business)

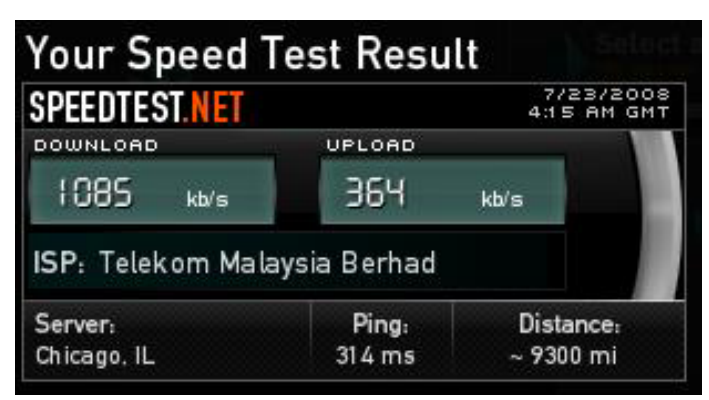

Figure 5. Line speed from VPN server to Malaysia (Source: http://www.speedtest.net/) 


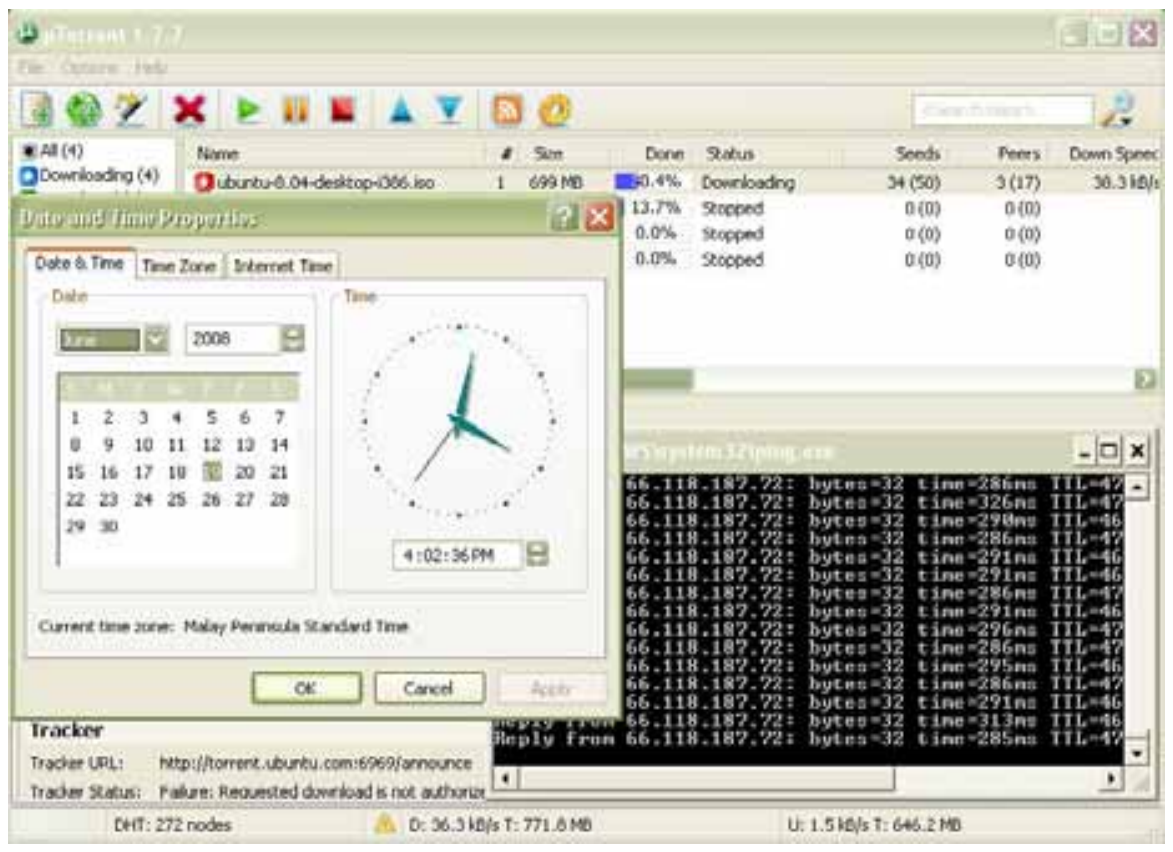

Figure 6. uTorrent downloading ubuntu without VPN



Figure 7. uTorrent downloading ubuntu with VPN 


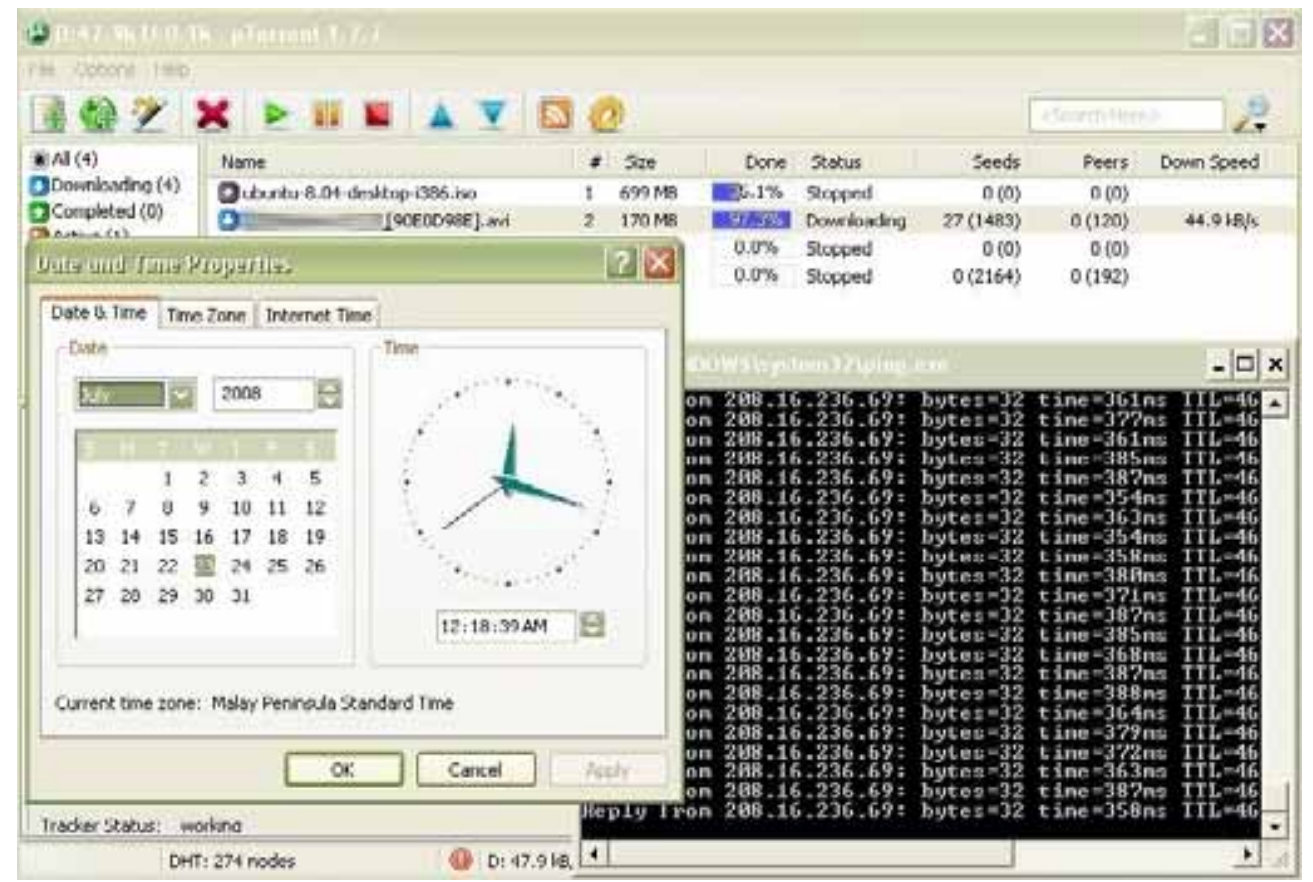

Figure 8. uTorrent downloading random files without VPN

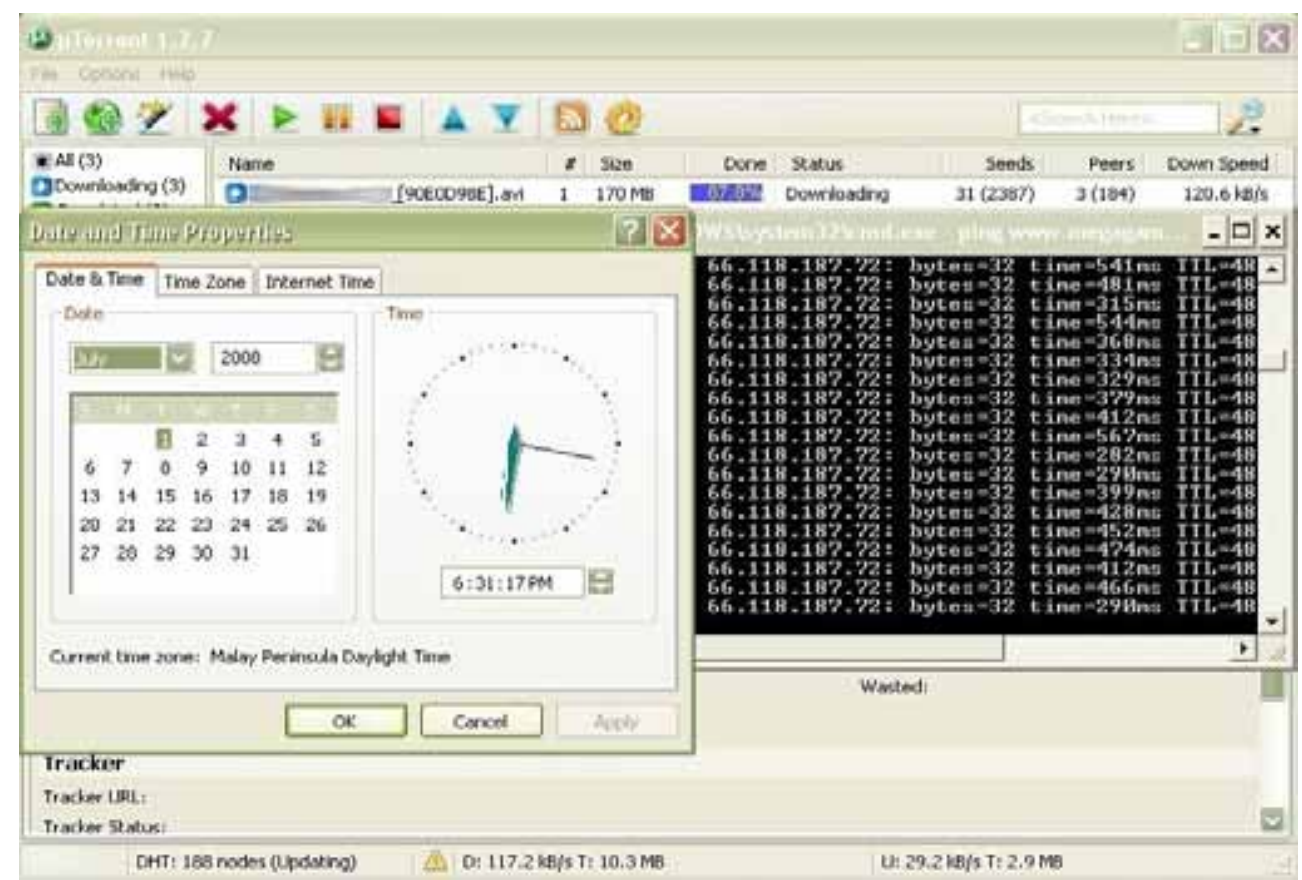

Figure 9. uTorrent downloading random files with VPN 


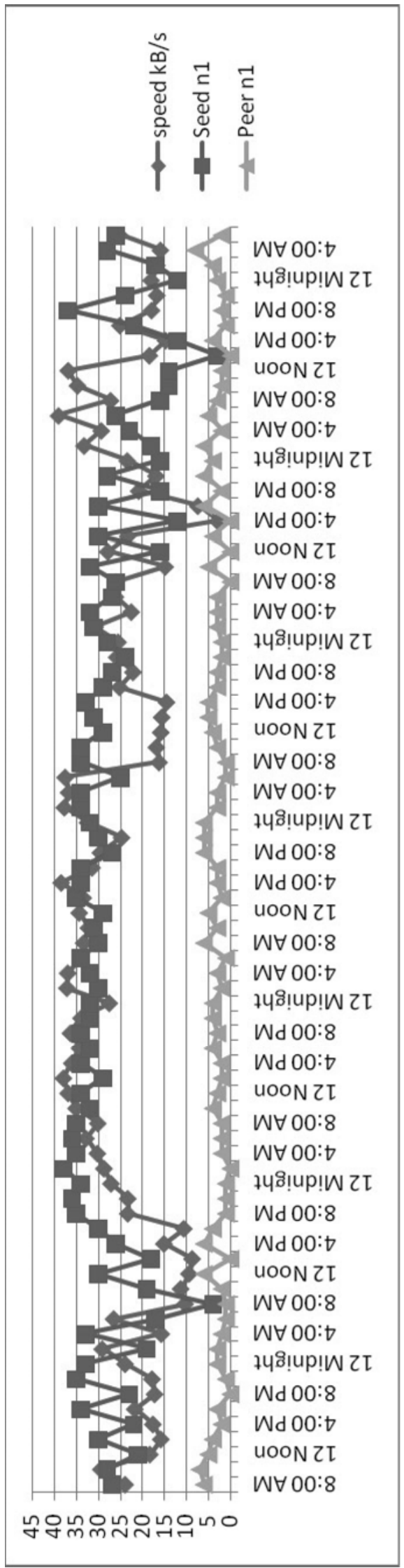

ป 




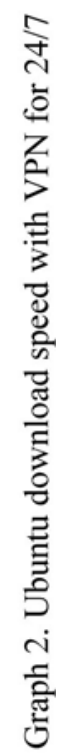




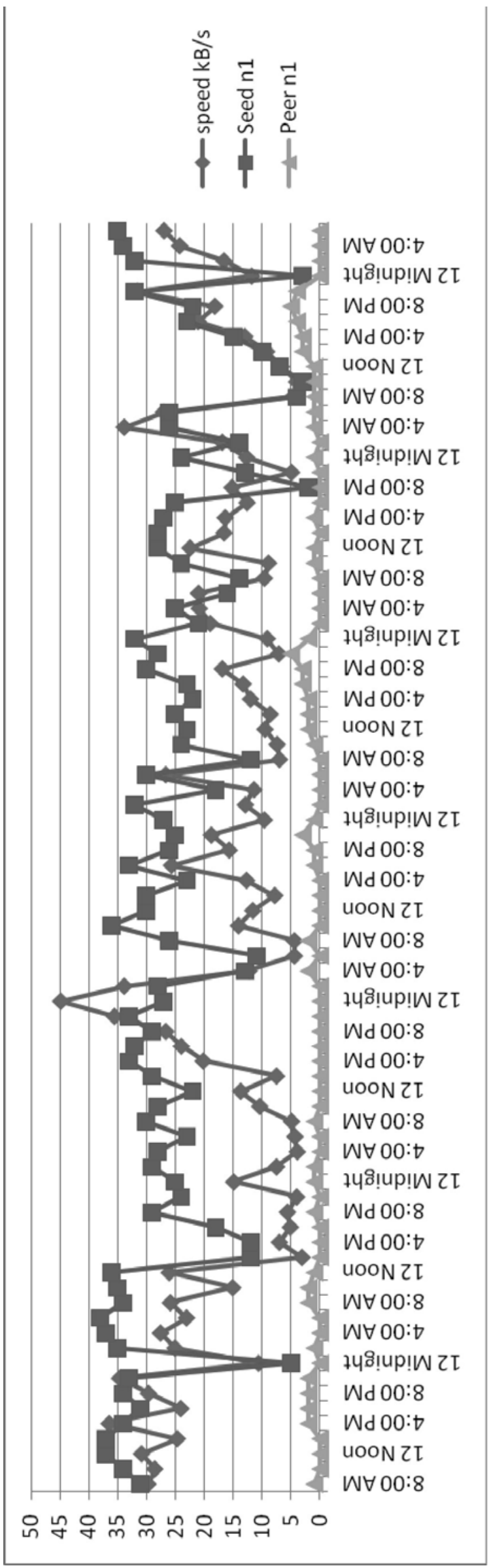

告 


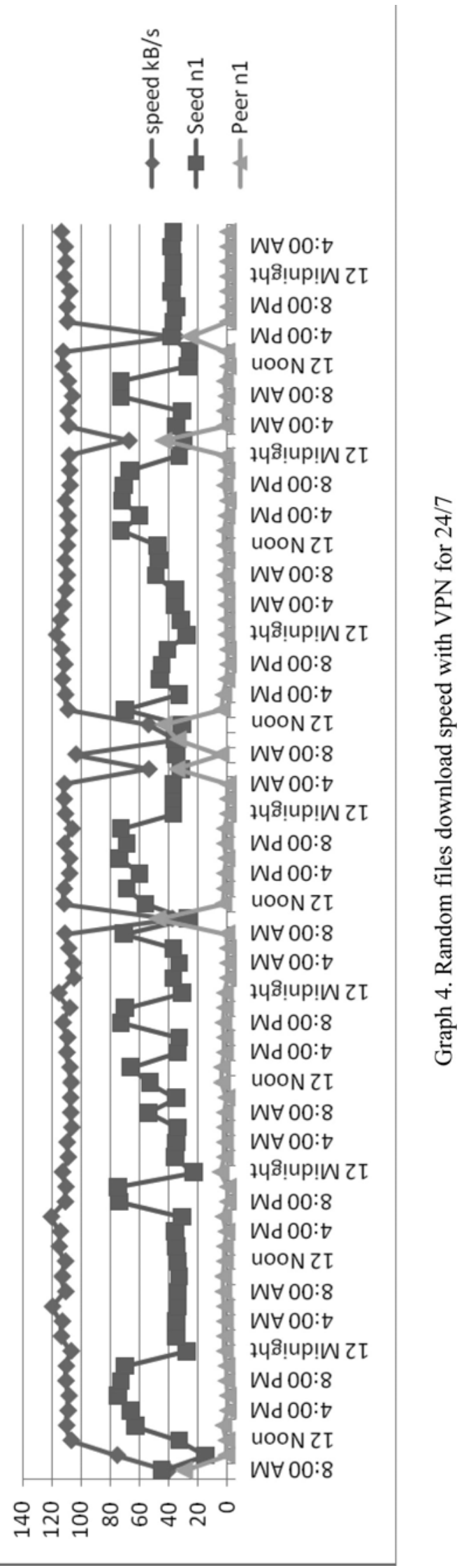






